room with 'dual facility' in this respect, in that individual benches would be about 1.8 metres in width to accommodate the appropriate stools for dentist and nurse for all disciplines (Fig. 1).

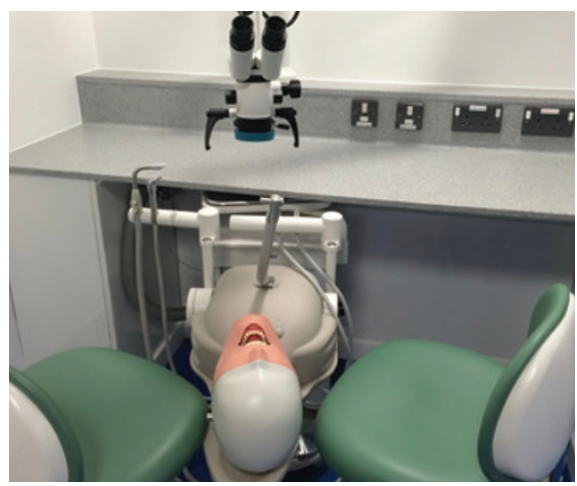

Fig. 1 The dual facility within the clinical skills simulation room at Morriston Hospital Swansea

Since its inception it has been used effectively to teach a spectrum of techniques from routine conservative procedures to more complex endodontics involving microscopy to teams and all including nurse participation.

This has led in the main to some resoundingly positive feedback from participants who have ranged from DF1 status new dentists to hardened practitioners with established 'bad habits'. I have no unequivocal evidence that such training leads to a better patient experience or a hike in quality but I remain convinced that 'on the balance of probabilities' dual training in such a fashion will have a positive impact on the task performance of dentist and close support nurse.

Are there many such facilities in the UK, and if not why not?

K. F. Marshall, by email DOI: 10.1038/sj.bdj.2016.118

\section{SAFEGUARDING CHILDREN}

\section{Was not brought}

Sir, safeguarding of children is an issue that should never be taken lightly.

With regards to the recognition of missed dental visits, the term 'did not attend' is commonly used, even with minors. A spin on this has been the introduction of the term "was not brought' as suggested by Powell et al. ${ }^{1}$ The terminology, which has been endorsed by consultant paediatric dentists from reputable institutes in the UK, is poignant as failure to attend for healthcare (without reason) is a breach of a child's fundamental rights and thus a child protection issue. The dental team should consider the implementation of this wording as a means of conveying the sincerity of missed appointments to parents.
Practice posters, emails and letters can provide parents with the reminder that the onus of a child's dental attendance lies with them.

Dentists can play an important role in detecting neglect and abuse but for this the child must be regularly attending. However, a lack of regular attendance can also raise an alarm bell.

\section{Chand, Northampton General Hospital}

1. Powell C, Appleton J V. Children and young people's missed health care appointments: reconceptualising 'Did Not Attend' to 'Was Not Brought' - a review of the evidence for Practice. J Res Nursing 2012; 17: 181-192.

DOI: 10.1038/sj.bdj.2016.119

\section{PHARMACOLOGY}

\section{Statins and persistent ulceration}

Sir, persistent oral ulceration is a commonly encountered problem in the dental practice. The cause may be difficult to identify and often requires expert input from an oral medicine/oral and maxillofacial specialist. With this letter we would like to remind the readers of the relationship between statins and persistent oral ulceration.

Statins are inhibitors of 3-hydroxy3-methylglutarylcoenzyme A (HMG-CoA) reductase that have revolutionisd the treatment of hypercholesterolemia. Their beneficial effects have been well documented. According to the British Heart Foundation, over 66 million statins prescriptions were written last year, a figure which has trebled in the past ten years. ${ }^{1}$

Adverse drug reactions (ADRs) to cardiovascular medication were outlined recently in the literature. ${ }^{2,3}$ The prevalence of oral manifestations of ADRs is not fully known, and the pathophysiological mechanisms for which these occur have yet to be fully elucidated; there have been reports in the literature associating oral ADRs to simvastatin use.

A 62-year-old gentleman recently presented to our clinic with a 12-month history of a recurrent keratotic lesion with areas of small ulceration on the right lateral border of the tongue, which became symptomatic when exposed to acidic or spicy foods. He took regular atorvastatin for hypercholesterolaemia; he was a non-smoker and recorded very occasional alcohol intake.

Histopathological analysis through an incisional biopsy suggested candidiasis with focal ulceration. A two week course of systemic fluconazole and topical nystatin were given; despite this the lesion persisted. Three months later, the patient presented with two additional healing aphthous-type ulcers in the buccal sulcus adjacent to the upper left canine and lower right second permanent molar.

Statins were suggested as a potential cause for the ulcerations and so were stopped. Six weeks later, the patient reported complete resolution of symptoms and no episodes of ulceration in this time had been noted.

Whilst many patients with oral ulceration have complex polypharmacy, statins are medications that could potentially be stopped without immediate complications and hence a potential causative link could be established or excluded.

D. J. Smith, M. Dillon, J. Russell,

A. Kanatas, Leeds

1. British Heart Foundation. Statin prescriptions in England rise again. 9 July 2014. Available at: https://www.bhf.org.uk/news-from-the-bhf/newsarchive/2014/july/statin-prescription (accessed February 2016).

2. Yuan A, Woo S B. Adverse drug events in the oral cavity. Oral Surg Oral Med Oral Pathol Oral Radiol 2015; 119: 35-47.

3. Kristensen M L, Christensen P M, Hallas J. The effect of statins on average survival in randomised trials, an analysis of end point postponement. BMJ Open 2015; 5: e007118.

DOI: 10.1038/sj.bdj.2016.120

\section{PERIODONTICS}

\section{Gingivitis and early labour}

Sir, I wished to share with your readership the story of a female patient who attended as a new patient to myself recently. She presented with generally good oral hygiene and minimal inflammation of the gingival tissues with no pocketing present and no loss of bone height radiographically, leading to a diagnosis of mild gingivitis.

During the course of my consultation she happened to mention that the birth of her daughter, now three years old, had taken place two weeks prematurely on the same day that she had attended an appointment for a 'scale and polish' with the hygienist.

The patient enquired as to whether I thought there could be a link. I answered that there has been a relationship established between periodontal disease and early labour due to inflammatory markers (prostaglandins) in the mother's blood but to date I do not know of any research that maintains a link between routine management of mild gingival conditions and labour induction.

I believe that this would be an important area for further study as if this lady's experience is not unique then, as a profession, we need to consider any potential risk of early labour induction in expectant mothers who receive any form of periodontal therapy in the final trimester of their pregnancy.

K. Bell, Bristol

DOI: 10.1038/sj.bdj.2016.121 